\title{
GLUCOSE TRANSPORTERS IN THE BLOOD-BRAIN BARRIER
}

\author{
Piret HuSSAR ${ }^{1}$, INDREK BENNO ${ }^{2}$, ÜLO HUSSAR ${ }^{1+}$ \\ ${ }^{1}$ Institute of Anatomy, Faculty of Medicine, University of Tartu, Estonia \\ ${ }^{2}$ Surgery Clinic, Tartu University Hospital, Estonia
}

\begin{abstract}
The central nervous system and peripheral nerves are guarded against free access from the outside by the blood-brain, blood-cerebrospinal fluid and blood-nerve barriers. The glucose transporter GLUT1 mediates the specific transfer of glucose across these barriers while GLUT3 is a high-affinity isoform of Type I glucose transporter expressed mostly in neurons where it is believed to be the main glucose transporter isoform. As for a long time it was an open question whether, GLUT1 and GLUT3 are present in the olfactory system, the aim of the present study was to give answers to these questions. In the study mucous membranes of the olfactory region of 20 male Wistar rats were studied by double immunofluorescence labeling. As the result of the study, the immunolocalization of glucose transporters GLUT1 in the endothelial cells of the olfactory mucosa and GLUT3 expression in olfactory receptor neurons were detected.
\end{abstract}

Key words: glucose transport, GLUT1, GLUT3, blood-brain barrier

\section{INTRODUCTION}

Glucose transporters are the membrane proteins that serve in the transfer of sugars across the cellular membranes $[1,2,11,12]$. Two types of glucose transporters have been identified: the SGLT family and the GLUT family. SGLT glucose transporters are sodium-dependent active transporters serving in the concentrative transport of sugars in the small intestine and the kidney. GLUT glucose transporters are the passive facilitated-diffusion transporters that trans- 
port sugars according to their concentration gradient. Previously it has been shown that GLUT1, an isoform of the GLUT family, is abundant in the cells of blood - tissue barriers $[10,13]$. The importance of GLUT1 was made evident by a mutation of GLUT1 that was shown to be responsible for seizures due to the decrease of the glucose level in the cerebrospinal fluid caused by defective glucose transport across the blood - brain barrier [9].

The olfactory system is a unique extension of the central nervous system as the sensory cells of the olfactory system, olfactory receptor neurons are embedded in the olfactory epithelium of the nasal mucosa, and protrude their dendrites to the lumen (3-5). Protein gene product 9.5 (PGP 9.5) is a useful marker for various types of neurons [14]. To clarify the olfactory mucosa antisera to tubulin and PGP 9.5 were used.

\section{MATERIAL AND METHODS}

In the experiment mucous membranes of the olfactory region of 20 four weeks old male Wistar rats were studied by double immunofluorescence labeling. Specimens were fixed with 1-3\% paraformaldehyde in phosphate-buffered saline (PBS) at $4{ }^{\circ} \mathrm{C}$ for 3-24 h; thereafter washed with PBS, infused with $20 \%$ sucrose in $0,1 \mathrm{M}$ sodium phosphate buffer, frozen in liquid nitrogen, and stored at $-80^{\circ} \mathrm{C}$ until use. Cryostat sections, $4-8 \mu \mathrm{m}$ thick, were cut and mounted on the glass slides coated with poly-L-lysine. Double-immunofluorescence labeling was carried out as described previously (Takata et al. 1990). Rabbit antiGLUT1, guinea pig anti-GLUT1 (raised by K. Takata, Gunma University, Japan), rabbit anti-chicken tubulin (from S. J. Singer, University of California at San Diego), rabbit anti-GLUT3 and mouse anti-PGP 9.5 served as primary antibodies. Fluorescein isothiocyanate-labeled donkey antiguinea pig immunoglobulin G (IgG), dichlorotriazinyl amino fluorescein-labeled and rhodamine red X-labeled donkey anti-rabbit IgG, and Cy3-labeled donkey anti-mouse IgG were used as secondary antibodies (Jackson Immunoresearch, West Grove, PA). Nuclei of the cells were counterstained with 4,6-diamidino-2-phenylindole (DAPI). Specimens were sequentially incubated with a mixture of the primary antibodies raised in different animal species, then with a mixture of fluorescence-labeled species-specific secondary antibodies. Immunolabeled samples were examined with AX-70 epifluorescence microscope (Olympus, Tokyo, Japan). 


\section{RESULTS}

The studies indicated the abundant presence of GLUT1 in the endothelial cells of olfactory mucosa while the cells of the olfactory epithelium were positive for PGP 9.5 (Figure 1). The upper cells of olfactory epithelium (cellulae neurosensoriae olfactoriae) stained srongly positive for GLUT3 (Figure 2). Antitubulin antibody strongly stained the apices of the olfactory epithelial cells as well as nerve fiber bundles emanating from the epithelium (Figures 3-4).

\section{DISCUSSION AND CONCLUSIONS}

The glucose transporter GLUT1 mediates the specific transfer of glucose across blood-brain, blood-cerebrospinal fluid and blood-nerve barrier barriers $[11,12]$.

The delivery of glucose from the blood to the brain involves its passage across the endothelial cells of the blood-brain barrier, which is mediated by the facilitative glucose transporter protein 1 and then across the neural cell membranes, which is mediated by GLUT 3 [6]. By Mantych et al. [8] it has been proved that the prominent localization of GLUT3 to mature neuronal processes suggests an essential role for this transporter in regulating fuel requirements for dendritic and axonal traffic, thereby mediating neurotransmission. However the question whether GLUT1 and GLUT3 are present in the olfactory system as well has been under discussion. GLUT1 1 and occludin may serve as a part of the machinery for the specific transfer of glucose in the olfactory system while preventing the non-specific entry of substances [7].

Our investigations showed the immunolocalization of glucose transporters GLUT1 in the endothelial cells of olfactory mucosa and GLUT3 expressing primarily in olfactory receptor neurons indicating to the pathway of glucose to cross the blood-brain barrier and enter neurons. The results also tshowed that tubulin acts as a marker for the nerve fibers in the olfactory mucosa and PGP9.5 serves as a marker both for the olfactory epithelium and nerve fibers. 


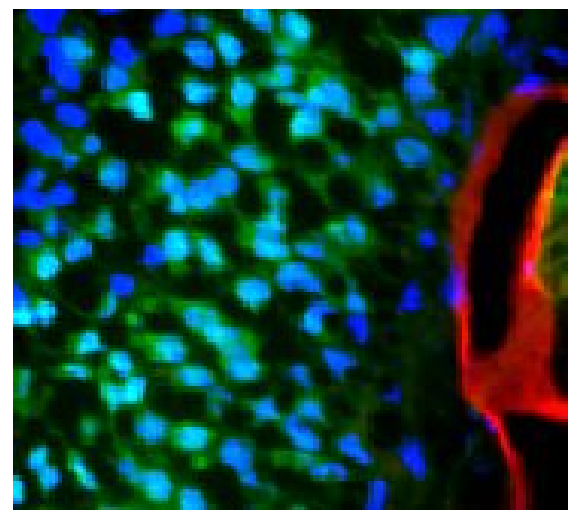

Figure 1. Immunofluorescence localization of GLUT1 and PGP9.5 in the olfactory mucosa. GLUT1 is abundant in the endothelial cells of the blood vessels and PGP 9.5 in the olfactory receptor neurons of the olfactory epithelium.

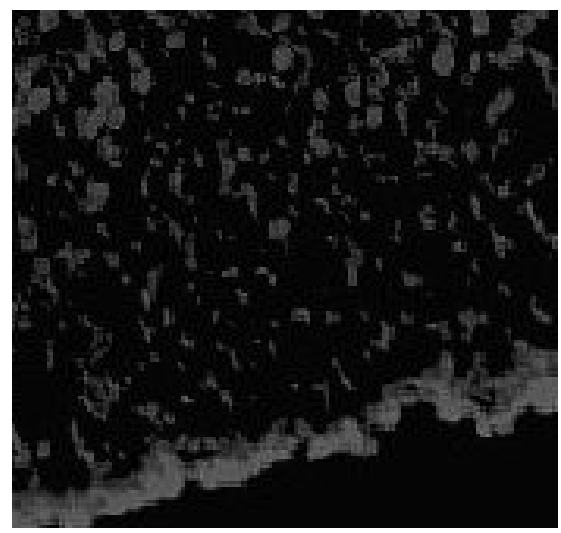

Figure 3. Immunofluorescence localization of tubulin and GLUT1 in the olfactory mucosa. The apices of the olfactory epithelial cells are strongly stained positive for tubulin.

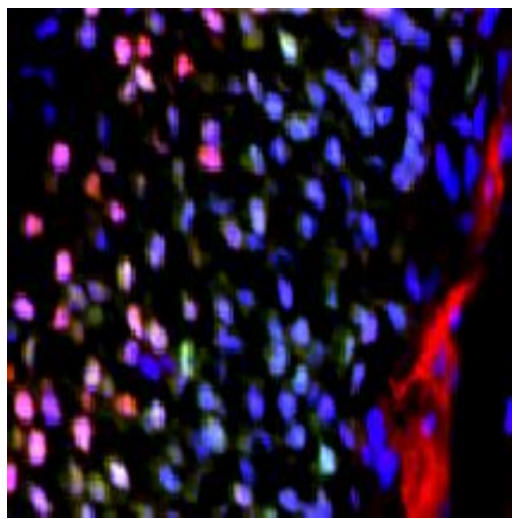

Figure 2. Immunofluorescence localization of GLUT3 and PGP9.5 in the olfactory mucosa. Note the upper cells of olfactory epithelium (cellulae neurosensoriae olfactoriae) stained positive for GLUT3.

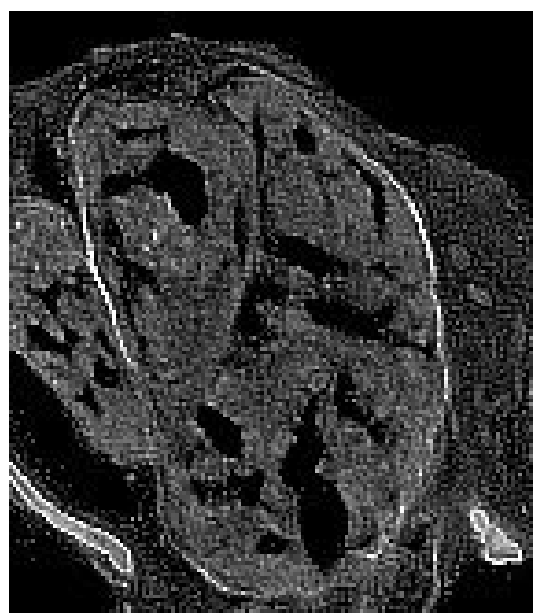

Figure 4. Immunofluorescence localization of tubulin and GLUT1 in the olfactory mucosa. The nerve fiber bundles emanating from the epithelium stained positive for tubulin while GLUT1 is localizing in the nerve fiber sheets and in the endothelial cells of the surrounding blood vessels. 


\section{ACKNOWLEDGEMENTS}

This work was supported in part by Grants-in-Aids for Scientific Research from the Ministry of Education, Science, Sports, and Culture of Japan. P.H. was the recipient of a Japanese Government (Monbusho) scholarship.

\section{REFERENCES}

1. Baldwin S. A. (1993). Mammalian passive glucose transporters: members of an ubiquitous family of active and passive transport proteins. Biochim Biophys Acta, 1154, 17-49.

2. Bell G. I., Burant C. F., Takeda J., Gould G. W. (1993). Structure and function of mammalian facilitative sugar transporters. J Biol Chem, 268, 19161-19164.

3. Doucette R. (1990). Glial influences on axonal growth in the primary olfactory system. Glia, 3, 433-449.

4. Farbman A. I. (1992). Cell Biology of Olfaction. Cambridge University Press, Cambridge.

5. Graiziadei P. P. C. (1973). The ultrastructure of vertebrate olfactory mucosa. In: Friedmann I. (ed.). The Ultrastructure of Sensory Organs. North-Holland, Amsterdam. 269-305.

6. Hou W. K., Xian Y.X., Zhang L., Lai H., Hou X. G., Xu Y.X., Yu T., Xu F. Y., Song J., Fu C. L., Zhang W. W., Chen L. Chin (2007). Influence of blood glucose on the expression of glucose trans-porter proteins 1 and 3 in the brain of diabetic rats. Chinese Medical Journal, 5, 120, 19, 1704-1709.

7. Hussar P., Tserentsoodol N., Koyama H., Yokoo-Sugawara M., Matsuzaki T., Takami S., Takata K. (2002). The glucose transporter GLUT1 and the tight junction protein occludin in nasal olfactory mucosa. Chem Senses, 27, 1, 7-11.

8. Mantych G. J., James D. E., Chung H. D., Devaskar S. U. (1992). Cellular localization and characterization of Glut 3 glucose transporter isoform in human brain. Endocrinology, 131, 3, 1270-1278.

9. Seidner G., Alvarez M. G., Yeh J., O’Driscoll K. R., Klepper J., Stump T. S., Wang D., Spinner N. B., Birnbaum M. J., De Vivo D. C. (1998). GLUT-1 deficiency syndrome caused by haploinsufficiency of the blood-brain barrier hexose carrier. Nature Genet, 18, 188-191.

10. Takata K., Kasahara T., Kasahara M., Ezaki O., Hirano H. (1990). Erythrocyte/ HepG2-type glucose transporter is concentrated in cells of blood - tissue barriers. Bioche. Biophys Res Commun, 173, 67-73.

11. Takata K., Kasahara M., Oka Y., Hirano, H. (1993). Mammalian sugar transporters: their localization and link to cellular functions. Acta Histochem Cytochem, 26, 165-178.

12. Takata K. (1996). Glucose transporters in the transepithelial transport of glucose. J Electron Microsc, 45, 275-284. 
13. Takata K., Hirano H., Kasahara M. (1997). Transport of glucose across the bloodtissue barriers. Int Rev Cytol, 172, 1-53.

14. Thompson R. F., Doran J. F., Jackson P., Phillon A. P., Rode, J. (1983). PGP9.5-a marker for vertebrate neurons and neuroendocrine cells. Brain Res, 278, 224-228.

Address for correspondence:

Piret Hussar, M.D., D.M.Sc

Institute of Anatomy

Faculty of Medicine

University of Tartu

Ravila 19, 50411 Tartu, Estonia

E-mail: piret.hussar@ut.ee 